\title{
DIAGNÓSTICO DIFERENCIAL DA LEUCEMIA LINFÓIDE AGUDA EM PACIENTES INFANTO-JUVENIS
}

\author{
Giselly Karitta Santana DANTAS ${ }^{1}$ \\ Lailanne Toledo Alves SILVA ${ }^{1}$ \\ Xisto Sena PASSOS ${ }^{2}$ \\ Cristiene Costa CARNEIRO ${ }^{3}$
}

\begin{abstract}
${ }^{1}$ Alunas do curso de Graduação em Biomedicina da Universidade Paulista, Campus Flamboyant, ${ }^{2}$ Professor Doutor em Medicina Tropical pela Universidade Federal de Goiás. Professor Titular do Curso de graduação em Biomedicina da Universidade Paulista - UNIP, Campus Goiânia Flamboyant, ${ }^{3}$ Professora Mestre em Biologia pela Universidade Federal de Goiás.
\end{abstract}

Endereço para correspondência do autor: Rua Borba Gato, quadra 32 lote 17, casa 02, Jardim Buriti Sereno III, Aparecida de Goiânia - GO, CEP. 74944-240. Cel. (62) 9289-2475. Email: profacristiene@gmail.com

Recebido em: 221/2014 - Aprovado em: 03/05/2015 - Disponibilizado em: 30/10/2015

\section{Resumo}

A leucemia linfóide aguda (LLA) é uma neoplasia hematológica heterogênea, caracterizada por uma disfunção das células tronco da medula óssea, que leva a uma proliferação clonal desordenada das células precursoras de origem linfóide. Ocorre principalmente na infância em crianças entre 2 e 5 anos de idade. Embora a causa da LLA seja desconhecida, é improvável que a transformação leucêmica seja resultante de um fato isolado, mas sim do acúmulo de múltiplos processos envolvendo interações complexas quanto à susceptibilidade do hospedeiro. Apesar de apresentar um alto percentual de cura, trata-se de uma doença rapidamente progressiva, que carece de urgência no tratamento para um melhor prognóstico e sobrevida dos pacientes, deve-se sempre obter um diagnostico precoce e diferencial, que deve conter características específicas da LLA, podendo assim, distingui-la das demais patologias que contém os sinais e sintomas compatíveis com os da LLA, como a mononucleose infecciosa e anemia aplásica. Isso só é possível com uma investigação clínica e laboratorial adequada, e com exames específicos, tais como, mielograma, imunofenotipagem, exames citoquímicos e citogenéticos. Estes exames permitem a classificação morfológica da LLA, além de diferenciar os diversos estágios de maturação das células e as anormalidades cromossômicas, possibilitando assim a qualificação dos pacientes em diferentes grupos de risco, sendo fundamental para determinar o tratamento adequado para cada caso. A demora do diagnóstico acarreta atraso no tratamento, o que diminui significantemente a sobrevida e/ou cura dos pacientes infanto-juvenis. Por este e outros pontos, o diagnostico diferencial tem seu lugar de destaque no prognostico da LLA.

Descritores: Diagnóstico, leucemia linfocítica aguda, câncer infantil, mielograma. 


\title{
DIFFERENTIAL DIAGNOSIS OF ACUTE LYMPHOID LEUKEMIA IN CHILDREN JUVENILE PATIENTS
}

\begin{abstract}
Acute lymphoid leukemia (ALL) is a heterogeneous hematological neoplasia, characterized by dysfunction of stem cells from the red bone marrow, leading to an uncontrolled clonal proliferation of the lymphoid precursor cells. Mainly occurs during childhood, between the ages of 2 thru 5 years old. Although the cause of ALL is unknown, it is unlikely that the leukemic transformation arises from an unusual fact, but rather the accumulation of multiple processes involving complex interactions between host susceptibility. Although presenting a high percentage of cure, it is a rapidly progressive disease that requires urgent treatment. For better prognosis and survival of patients, it should always be obtained an early and differential diagnosis, which must contain specific features of ALL and can thus distinguish it from other diseases that presents signs and symptoms compatible to ALL. This is only possible with an adequate clinical and laboratory research and specific tests such as complete, myelogram, immunophenotyping, cytochemical and cytogenetic tests. This tests allows the morphological classification of ALL, in addition to differentiate the various stages of cell maturation and chromosome abnormalities, thus enabling the qualification of patients in different risk groups which is fundamental to determine the appropriate treatment for each case. The delay in diagnosis leads to delayed treatment, which significantly decreases the survival and/or cure of children and young patients. For this and other points, the differential diagnosis has its place in the prognosis of ALL.
\end{abstract}

Descriptors: Diagnosis, acute lymphocytic leukemia, childhood cancer, myelogram.

\section{Introdução}

A leucemia linfóide aguda (LLA)

é uma neoplasia hematológica heterogênea, caracterizada por uma disfunção das células tronco da medula óssea vermelha que leva a proliferação clonal desordenada de células precursoras de origem linfóide que passam a circular no sangue na sua forma imatura $(1,2)$. Esse comprometimento dos linfoblastos na medula óssea vermelha, também dificulta a produção normal de outros glóbulos brancos, vermelhos e plaquetas, levando a substituição dos elementos da medula óssea vermelha pelas células leucêmicas (3).

A LLA é uma doença de início abrupto e evolução rápida, porém, potencialmente curável, apresentando ampla diversidade de aspectos clínicos e biológicos (4). Existem subtipos diferentes de linfócitos, e, portanto tipos diferentes de leucemia, que são classificados de acordo com a célula envolvida, a duração e o caráter da doença. Dentro dessa enfermidade, as células de LLA pré-B são responsáveis por mais ou menos $85 \%$ dos casos, 
enquanto $15 \%$ são representados por células pré-T(2).

$\mathrm{Na}$ população infantil, estima-se uma incidência anual de cerca de 200 mil novos casos de câncer em todo o mundo, sendo a leucemia o tipo mais comum. No Brasil, de 10 - 15 novos casos de câncer abaixo de 15 anos, 4 são LLA (27\% à 40\%). Tal leucemia pode atingir tanto adultos jovens como crianças, sendo o câncer infantil mais frequente, com pico de incidência entre os 2 e 5 anos de idade. A LLA é mais comum em pacientes do sexo masculino e raça branca. $\mathrm{O}$ percentual de cura da LLA é em torno de $80 \%$, avanço esse decorrente da melhora no diagnóstico, identificação de fatores prognósticos e utilização de tratamentos adaptados ao grupo de risco de cada paciente (5).

Já nos adultos, a LLA representa $20 \%$ das leucemias agudas com sobrevida global (tempo decorrido entre o diagnóstico e o óbito), prolongada em torno de $30 \%$ à $40 \%$. A incidência volta a aumentar após os 60 anos, com um pior prognóstico clínico (6).

A etiologia da leucemia ainda é considerada incerta, mas muitos estudos apontam para fatores causais como infecção viral, exposição à radiação e exposição química. A LLA evoluiu de uma neoplasia mal definida e intratável na metade do século passado para uma doença bem relatada na literatura e com resultados relevantes de cura no início deste século (7).

O diagnóstico feito por testes que apresentam eficácia relevante, isto é, testes com especificidade e sensibilidade de aproximadamente $100 \%$, evitam resultados falso-negativos e garantem um bom prognóstico ao paciente (8). Decorrente da preocupação em assegurar os resultados emitidos realiza-se uma série de exames confirmatórios e comprobatórios dessa patologia.

Os sintomas observados quando há acometimento por LLA, são pouco específicos e podem ser confundidos com várias patologias comuns da infância, como a mononucleose infecciosa, coqueluche, doenças virais, processos inflamatórios, artrite reumatóide juvenil, artralgia, fadiga e fraqueza decorrentes de anemia, febre e/ou outros sintomas de infecção, perda de peso e/ou de apetite, sangramentos, dores nas articulações e ossos, linfonodomegalia, esplenomegalia e hepatomegalia. O diagnóstico da LLA é feito basicamente por exame físico e exames laboratoriais. Os principais exames utilizados para diagnosticar a LLA são, primeiramente o Hemograma 
e Mielograma, seguido de exames confirmatórios

como

Imunofenotipagem, Cariótipo e Análise da presença de Translocações (3).

O diagnóstico precoce e o pronto início do tratamento têm importante papel na redução da mortalidade $\mathrm{e}$ morbidade em relação à doença. Percebendo a importância desse assunto, o presente trabalho foi desenvolvido tendo como objetivo apontar os avanços e perspectivas no diagnóstico diferencial da LLA em pacientes infanto-juvenis.

\section{Revisão da Literatura}

Trata-se de estudo de revisão narrativa de literatura. A busca bibliográfica foi realizada na base eletrônica de dados disponível na SciELO, LILACS, PubMed, Google acadêmico e NCBI, utilizando como descritores os termos, leucemia linfocítica aguda, diagnóstico, câncer infantil, mielograma e outros. Foram utilizados artigos publicados no período de 2010 a 2014, nos idiomas português, inglês e espanhol. O presente estudo implicou-se no levantamento de dados de várias fontes de pesquisa, seguindo métodos rigorosos, a partir da leitura atenta e interpretativa, a fim de levantar o maior número de dados, atualizados e fidedignos.

A revisão da literatura foi dividida nos seguintes subtítulos: Sinais e Sintomas Clínicos; Hemograma, Mielograma e Morfologia Celular; Citoquímica; Citogenética; e Imunofenotipagem.

Sinais e Sintomas Clínicos

O diagnóstico da LLA é um processo complexo, e muitas são as variáveis que parecem influenciá-lo, por possuir sinais e sintomas muito inespecíficos semelhantes ao de outras patologias benignas comuns da idade, como mononucleose infecciosa, coqueluche, determinadas doenças virais, processos inflamatórios, variados graus de anemia, artrite reumatóide juvenil, artralgia, entre outras (5). Por consequência desta vasta lista, é possível não se detectar precocemente a LLA ao exame físico, portanto, são necessários exames laboratoriais confirmatórios (9).

O diagnóstico da LLA é estabelecido pelos sinais e sintomas apresentados pelo paciente em conjunto com os achados laboratoriais (10). Os sinais e sintomas apresentados em decorrência da baixa produção de 
células sanguíneas são: anemia, palidez progressiva, cansaço a mínimos esforços e sonolência ocorrem pela diminuição de hemoglobina no sangue. Hematomas, petéquias e sangramentos prolongados das mucosas, ocorrem pela diminuição das plaquetas. Infecções e hipertermia frequentes ocorrem pela diminuição dos leucócitos funcionais (11).

Aumento dos gânglios linfáticos pelo acúmulo de linfoblastos leucêmicos no sistema linfático. Cefaléia e vômitos, causados pelo acúmulo de células leucêmicas no líquido céfalo raquidiano. Com a evolução da doença, podem ocorrer, ainda, acometimento ocular, testicular, nódulos subcutâneos, aumento das glândulas salivares, priapismo e síndromes compressivas medulares (6). Pacientes com estas manifestações, frequentemente têm hemogramas iniciais normais, o que pode contribuir para o atraso do diagnóstico (9).

A confirmação do diagnóstico é feita pela realização do hemograma e mielograma, sendo complementados pelos exames de imunofenotipagem, citogenética e biologia molecular, estes últimos fundamentais para a escolha do esquema terapêutico (12).
Hemograma, Mielograma e Morfologia Celular

O diagnóstico e a classificação das leucemias agudas baseiam-se, em grande parte, na análise morfológica das células neoplásicas. O diagnóstico é feito primeiramente por hemograma (sangue periférico) e mielograma (medula óssea), além das técnicas de citomorfologia, imunofenotipagem e citogenética realizadas para complementação (13).

O hemograma é o exame inicial, que avalia a quantidade a e qualidade das células sanguíneas. Normalmente é requerido para diagnosticar ou controlar a evolução de uma doença. $\mathrm{O}$ hemograma completo é constituído por contagem de plaquetas, leucograma e eritrograma (5).

O hemograma pode revelar anemia normocítica e normocrômica e trombocitopenia. A contagem de leucócitos está ocasionalmente muito alta, mas frequentemente normal ou diminuída. Os blastos são raros ou ausentes em pacientes leucopênicos, mas em casos de leucocitose podem ser numerosos, chegando a constituir maioria (8).

O Mielograma é o exame que analisa e quantifica os componentes da 
medula óssea. É realizado através de uma punção feita no esterno, ossos do quadril e na tíbia (13).

O diagnóstico de LLA é estabelecido quando $25 \%$ ou mais das células nucleadas da medula óssea são linfoblastos. Em quase todos os pacientes com LLA, as descrições do mielograma incluem medula óssea hipercelular com intensa infiltração por linfoblastos com substituição dos espaços adiposos e elementos medulares normais por células leucêmicas, com precursores mielóides e eritróides residuais de aspecto normal e megacariócitos diminuídos ou ausentes (14).

A classificação morfológica foi desenvolvida nos anos 70, para as leucemias agudas pelo grupo FrancêsAmericano-Britânico (FAB), com base no diâmetro das células, protuberância dos nucléolos e quantidade de citoplasma. O grupo dividiu a LLA em três subtipos morfológicos (L1, L2 e L3), sendo o subtipo L1 o mais comum em crianças. Nesse subtipo, os linfoblastos apresentam-se pequenos, com contorno nuclear regular, sem nucléolos, com pouco citoplasma, sem basofilia. No subtipo L2 os blastos (10\% dos casos), apresentam células de tamanhos diversos cujo citoplasma varia de tamanho e basofilia, podendo apresentar nucléolos e irregularidades de contorno. Por último no subtipo L3, os pacientes apresentam células grandes com nucléolos, basofilia citoplasmática e vacúolos, com o imunofenótipo $B$, sendo considerada a forma leucêmica do linfócito de Burkitt, com blastos mais raros, compreendendo de 1 a $2 \%$ das $\operatorname{LLA}(2,4)$.

\section{Citoquímica}

As reações citoquímicas auxiliam na diferenciação entre LLA e LMA. Os linfoblastos revelam atividade nuclear inespecífica, coloração alterada e forma de anéis concêntricos e vacúolos. Uma reação negativa diante da aplicação citoquímica é sugestiva de LLA, enquanto a positiva caracteriza uma LMA. As reações citoquímicas mais utilizadas incluem Sudan-Black B (SBB), mieloperoxidase (MPO), reação de Ácido Periódico de Schiff (PAS) e esterases não-específicas (NSE) (11).

O SBB cora lipídios, gorduras neutras, fosfolipídios e ésteres. É específico para células mielóides, geralmente acompanha a MPO. A partir dos promielócitos a coloração é fortemente positiva, aumentando com a maturação $(2,7)$. 
A MPO é específica da linhagem mielóide, localiza-se em grânulos azurófilos primários de neutrófilos e monócitos. As células granulocíticas expressam positividade crescente conforme maturação, enquanto as monocíticas expressam granulações menos intensas, apresentando grânulos finos, dispersos por toda célula. Pode também ser positiva em grânulos específicos de eosinófilos e basófilos. Os blastos da linhagem mielóide demonstram atividade em áreas como retículo endoplasmático e região de Golgi. A MPO promove então a clivagem do peróxido de hidrogênio, liberando oxigênio, que reage com a benzidina, formando um composto de cor castanho esverdeado. Esta reação é útil na diferenciação entre leucemias mielóide e linfóide aguda (4).

O PAS é importante na citoquímica de carboidratos, pois oxida compostos com grupos hidroxilas livres, que combinam com o reativo de Schiff obtendo a coloração rósea. Os mieloblastos possuem pouco glicogênio, aumentando seu teor a partir do promielócito e consequentemente sua positividade, evidenciando um padrão difuso em todo o citoplasma. Esta reação é intensamente positiva em 40 $70 \%$ das LLAs, com formação de anéis, unipolar ou blocos (13).

As NSE são enzimas que hidrolisam ligações éster de alcoóis, fenóis e naftóis. As reações positivas podem ser encontradas na linhagem granulocítica, nos promonócitos e nos monócitos maduros. As esterases não específicas são usadas para identificar células de origem monocítica, sendo inibida pelo fluoreto de sódio. A reação é fortemente positiva nos monócitos, negativa em monoblastos e fracamente positiva ou negativa nos granulócitos (15).

\section{Citogenética}

Dentre os casos de LLA, apenas $5 \%$ estão relacionados com predisposições genéticas, como Síndrome de Down, Síndrome de Bloom, Ataxia-telangiectasia, Síndrome de quebra de Nijmegen ou radiação ionizante (14).

A análise cromossômica (cariótipo) das doenças hematológicas é de extrema relevância para que seja feito um diagnóstico mais refinado da malignidade das LLAs e do mecanismo de ação da patologia assim como a importância biológica (16).

Um dos mecanismos envolvidos na leucemogênese consiste na alteração 
de genes envolvidos com o controle do ciclo celular. Dentre esses estão os oncogênes, que codificam, em geral, proteínas envolvidas com a reprodução desordenada de células e com a resistência a apoptose (16). Mutações em sequências de DNA podem causar grandes variações estruturais no genoma, promovendo a ativação de oncogênes ou a inativação de genes com atividade supressora de tumor (2).

A presença de alterações cromossômicas tem grande importância no prognóstico. Dentre elas, as translocações apresentam grande efeito, pois permitem que oncogênes, antes não ativos, possam ser ativados por promotores depois da fusão com outros genes. Além disso, variações em sequência podem alterar genes que codificam enzimas metabolizadoras de drogas, transportadores, receptores $\mathrm{e}$ alvo de fármacos, resultando em diferenças farmacológicas durante $\mathrm{o}$ tratamento quimioterápico $(4,11)$.

As alterações cromossômicas mais comuns, como hiperdiploidia com mais de 50 cromossomos nos blastos leucêmicos e a translocação dos cromossomos 12 e 21 com a fusão do gene TEL-AML1, correspondem a aproximadamente $50 \%$ dos casos de LLA em crianças e representam um bom prognóstico. Enquanto maus prognósticos estão associados à presença da translocação $t(9 ; 22)$, conhecida como Cromossomo Filadélfia $(\mathrm{Ph})$, com fusão gênica BCR-ABL, que codifica uma proteína quimérica com atividade tirosina quinase muito elevada, resultando em proliferação celular e leucemogênese, porém está presente em apenas $2 \%$ à $4 \%$ dos casos pediátricos e diminui em 20\% a sobrevida em cinco anos, e a translocação $\mathrm{t}(4 ; 11)$ com fusão do gene MLL-AF4 (16).

A combinação de citogenética com outros métodos moleculares tem como principal objetivo identificar anomalias cromossômicas em casos de leucemia aguda em criança (6).

\section{Imunofenotipagem}

A imunofenotipagem das doenças malignas do sistema hematopoiético fundamenta-se na investigação da presença ou ausência de antígenos encontrados na superfície ou no citoplasma celular. Cabe ressaltar que o diagnóstico de uma doença maligna hematopoiética não se baseia em um único marcador específico, mas essa análise deve ser realizada utilizando-se um painel de Anticorpos Monoclonais, 
adequadamente selecionado para a determinação das linhagens celulares B, $\mathrm{T}$, NK e de células mielomonocíticas. Ainda, a imunofenotipagem visa à determinação do grau de diferenciação celular (imatura ou madura), a expressão antigênica aberrante nas populações celulares malignas e a presença ou não de clonalidade (apenas nas células da linhagem linfóide B) $(2,3)$.

A classificação por imunofenotipagem utiliza um painel de associações lineares monoclonais pelo qual a maioria dos casos de LLA pode ser subdividida em subgrupos de precursores de célula B e subgrupos de precursores de célula T. Os precursores de célula $B$ são ainda subdivididos em BI ou LLA pró-B, BII ou LLA comum, BIII ou LLA pré-B e BIV ou LLA-B madura, e as células $\mathrm{T}$ são divididas, atualmente, dentro de quatro subtipos: pró-T, pré-T, cortical e maduro (3).

A técnica da imunofenotipagem possibilita a classificação das LLAs fazendo a análise das características imunofenotípicas dos linfoblastos, além da detecção da leucemia através deste método é possível classificar o grau de diferenciação das células leucêmicas possibilitando um diagnóstico com maior especificidade (11).
A imunofenotipagem, realizada com a técnica de citometria de fluxo (CMF), é útil tanto no diagnóstico, classificação, prognóstico, estadiamento, monitoramento, como na caracterização fenotípica das células hematopoiéticas patológicas (2).

\section{Tratamento}

A quimioterapia é uma técnica de tratamento onde se utilizam compostos químicos isoladamente ou em combinações (poliquimioterapia) a fim de tratar tumores malignos. Constitui uma maneira de se combater o câncer por apresentar uma abordagem sistêmica, tornando possível a cura de leucemias. $\mathrm{O}$ tratamento por quimioterápicos na leucemia é realizado em 4 fases visando a se alcançar a remissão completa. São estas: indução da remissão, que requer a hospitalização do indivíduo; consolidação, em que substâncias não utilizadas anteriormente são empregadas; reindução, na qual são repetidas as drogas utilizadas na fase de indução da remissão e, por fim, a fase de manutenção, utilizando um tratamento mais leve e contínuo por vários meses podendo chegar a 1 ou 2 anos dependendo do tipo de leucemia (17). 
A utilização da estratégia terapêutica denominada de transplante de medula óssea (TMO) tem crescido muito nos últimos anos. Como parte do procedimento de TMO, seja ele utilizando células do próprio paciente (autólogo) ou de um doador previamente selecionado por testes de compatibilidade (alogênico). A medula óssea ou o sangue periférico são utilizados como fonte de células-tronco hematopoiéticas que irão promover a recuperação hematológica após o tratamento com quimioterapia em altas doses (18).

Os progressos no tratamento se devem a vários fatores, entre eles a disponibilidade cada vez maior de medidas de suporte e o aparecimento de antineoplásicos efetivos e suas combinações em muitos ciclos, como a combinação de Ciclofosfamida (agente citotóxico alquilante, que impede a divisao celular), Vincristina (alcalóide citotóxico natural e antimitótico específico para as fases $\mathrm{M}$ e $\mathrm{S}$ do ciclo celular) e Doxorrubicina (antibiótico citotóxico antraciclínico que intercala a dupla hélice do DNA, formando complexo ternário com topoisomerase II e DNA, desencadeando a apoptose celular), são medicamentos com poder emetogênico considerável, variando de alto a baixo, de acordo com a dose administrada. Indubitavelmente, o progresso extraordinário obtido nos últimos 30 anos no tratamento da LLA infantil está associado ao trabalho desenvolvido pelos diferentes grupos cooperativos envolvidos na sistematização dos protocolos terapêuticos, permitindo que se obtenham conclusões estatisticamente significativas em um menor período de tempo (19).

\section{Discussão}

As LLAs compreendem um grupo de neoplasias heterogêneas, essa heterogeniedade é resultante do fato da LLA se desenvolver em qualquer etapa da diferenciação linfóide normal, caracterizadas pela proliferação clonal e acúmulo na medula óssea de células hematopoiéticas imaturas denominadas linfoblastos, os quais ocupam o ambiente medular inibindo $\mathrm{o}$ crescimento e maturação normal dos precursores eritróides, mielóides e megacariocitários. Embora a LLA possa ocorrer em qualquer idade, sua incidência é maior entre crianças de 2 a 5 anos de idade (20). Perez-Vera et al., em 2001, encontraram $78 \%$ dos pacientes nessa faixa etária (22), 
enquanto Udayakumar et al., em 2007, observou em $76 \%$, de 2 a 5 anos de idade (23). Um estudo feito por Lichtvan em 2007, observou que $78 \%$ dos pacientes apresentavam entre um e dez anos de idade, sendo $38 \%$ entre dois e cinco anos (24).

É predominante nas pessoas de cor branca e do sexo masculino. Esse fato foi observado em estudos feitos por Swaminathan et al., em 2008 (25). Estudos feitos por Ries et al., em 1999 e Linet et al., em 2003 nos EUA, observaram um número crescente de ocorrências de LLA em crianças da cor branca em comparação com as crianças de cor negra (26). Concordando com esses autores, Linabery e Ross em 2008, observaram essa mesma tendência em relação ao sexo e cor (27).

O diagnóstico deve sempre ser confirmado pelo exame citológico de aspirado da medula óssea, exame obrigatório na suspeita de LLA, pois é o exame que melhor demonstra a presença dos blastos nesse órgão. A organização mundial da saúde (OMS) considera uma contagem de células blásticas na medula óssea maior que $20 \%$ o suficiente para o diagnóstico dessa doença (9). O grupo $\mathrm{FAB}$, no entanto, considera um valor mínimo de $30 \%$ (2). Um estudo feito por Ma et al., em 1997, observou que $20 \%$ dos pacientes apresentavam leucometria acima de 50.000/ $\mu \mathrm{L}$ (28). Outro estudo realizado na Inglaterra por Chessels et al., em 2003, mostrou que $21 \%$ dos pacientes apresentavam valores acima de $50.000 / \mu \mathrm{L}$ (29). O que, de acordo com o World Health Organization Classification of Tumours (WHO), diz que a leucometria em pacientes com leucemia linfoblástica aguda pode se apresentar diminuída, normal ou bastante elevada (14).

No hemograma podemos avaliar variados graus de anemia (normocítica e normocrômica), neutropenia e trombocitopenia. A contagem de leucócitos encontra-se habitualmente elevada com nítido predomínio de blastos (4).

Em relato feito por Margolin, Pizzo e Poplack em 2005, 80\% dos pacientes estudados com LLA apresentaram valores de hemoglobina menor que $10 \mathrm{~g} / \mathrm{dL}$ (30). Swaminathan et al., em 2008, observaram que $75 \%$ das crianças abaixo de dez anos, apresentavam hemoglobina abaixo de $10 \mathrm{~g} / \mathrm{dL}$, mesmo em concentrações baixas de hemoglobina, a anemia é normocítica e normocrômica, e a contagem de reticulócitos é reduzida. A trombocitopenia está presente na 
maioria dos pacientes, sendo a contagem de plaquetas menor que $100.000 / \mu \mathrm{L}$ em aproximadamente $75 \%$ dos casos (25).

Quanto à imunofenotipagem, $80 \%$ dos casos expressam marcadores de células B e $20 \%$ de células T. Tratase de uma técnica confirmatória para leucemias indiferenciadas, com morfologia inespecífica e citoquímica negativa (21). Rego et al., em 1997, em ribeirão preto, avaliaram 153 crianças, sendo $82 \%$ LLA de linhagem B (31). Dworzak et al., em 2003, na Áustria, encontraram LLA de linhagem B em $87 \%$ dos casos (32), e Udayakumar et al., em 2007, $83 \%$, sendo $7 \%$ de linhagem T (23). Leite et al., em 2007, estudaram 108 pacientes pediátricos, desses, $81 \%$ eram de linhagem B e $19 \%$ LLA-T. Farias e Castro, em 2004, observou um percentual de $80 \%$ de LLA-B e $20 \%$ de LLA -T (33).

Após a descoberta por Casperson et al., em 1970 (34), e Seabright em 1971 (35), das técnicas de bandeamento, os estudos citogenéticos nas LLAs demonstraram que poderiam ser encontradas cerca de 50\% de anomalias cromossômicas. De acordo com o estudo realizado por Kowalczyk et al., em 2010, 13,5\% dos pacientes apresentaram índice mitótico nulo, enquanto que em $24,7 \%$ foi observado cariótipo normal (34). Silva et al., em 2002, observaram que $92 \%$ dos pacientes apresentaram algum tipo de anormalidade cromossômica, $7 \%$ apresentaram cariótipo normal e 1\% das amostras analisadas apresentaram ausência de crescimento celular (35). Pérez-Vera et al., em 2001, encontraram, através da análise citogenética, $22 \%$ dos pacientes com cariótipo normal e 74\% com alguma alteração citogenética (22). Segundo a literatura, portadores de LLA com citogenética normal apresentam melhor prognóstico com $90 \%$ de sobrevida em cinco anos, e $70 \%$ à $92 \%$ dos portadores de LLA apresentavam alterações cariotípicas/citogenéticas.

O prognóstico dos pacientes com LLA depende de uma série de fatores que incluem o sexo, idade, leucometria inicial, anormalidades citogenéticas, imunofenótipo e a resposta ao tratamento, os quais permitem separar os pacientes em diferentes grupos de risco (baixo, intermediário e alto risco), levando a diferentes estratégias de tratamento, como a quimioterapia, radioterapia e transplante de medula óssea (TMO) (19).

$\mathrm{Na}$ maioria dos centros $\mathrm{O}$ tratamento da LLA envolve a 
quimioterapia intensiva em curto prazo. A radiação pode ser utilizada em pacientes que mostram evidência de acometimento do sistema nervoso central ou leucemia testicular (19).

\section{Conclusão}

Embora a leucemia linfocítica aguda possa ocorrer em qualquer idade, sua incidência é maior entre crianças de 2 a 5 anos. Como sua causa é desconhecida, é improvável que seja resultante de um evento isolado, mas sim do acúmulo de múltiplos processos. A LLA é uma doença de evolução rápida, podendo levar ao óbito em poucos meses, por isso, necessita de um diagnóstico e tratamento precoce. Sendo assim, um diagnóstico seguro da doença só pode ser feito depois de um exame clínico minucioso seguido de exames específicos. Os avanços técnicos no diagnostico diferencial tem contribuído para um melhor prognóstico e tratamento, tornando cada vez mais eficazes para a cura, menos agressivos e menos invasivos, baseado nas características individuais de cada criança. Portanto, é inquestionável a relevância e necessidade da utilização de exames que apresentem um maior grau de sensibilidade e especificidade para o diagnóstico diferencial da LLA, possibilitando uma intervenção precoce no tratamento e proporcionando ao paciente uma maior qualidade de vida.

\section{Referências}

1. Morais EF De, Lira JADS, Macedo RADP, Santos KS Dos, Elias CTV, Morais MDLSDA. Oral manifestations resulting from chemotherapy in children with acute lymphoblastic leukemia. Braz J Otorhinolaryngol [Internet]. 2014;80(1):78-85. Acesso em: http://www.ncbi.nlm.nih.gov/pub med/24626896

2. Vasconcelos G, Alves DA. Caracterização Hematológica e Imunofenotípica em Pacientes com Leucemia Linfoblástica Aguda Caracterização Hematológica e Imunofenotípica em Pacientes com Leucemia Linfoblástica Aguda. 2012.

3. I EPN, I HTM, Bárbara E, Fonseca A, Ii T, Assunção C, et al. Immunophenotypic characterization of acute leukemia at a public oncology reference center in Maranhão , northeastern Brazil Caracterização imunofenotípica 
das leucemias agudas em um centro oncológico de referência público no Maranhão, Nordeste do Brasil. 2011;129(6):392-401.

4. Fadel AP. Investigação Laboratorial de LLA. 2014.

5. Ferreira JD, Couto AC, Alves LC, Koifman S. Exposições ambientais e leucemias na infância no Brasil: uma análise exploratória de sua associação. 2012;477-92.

6. Bueno D. Marcelino Oliveira Cazé , Denise Bueno , Maria Elisa Ferreira dos Santos. 2011;(4).

7. Ganazza NA. ESTUDO DE DOENÇA RESIDUAL MÍNIMA EM LEUCEMIA LINFÓIDE AGUDA DA CRIANÇA E DO.

8. Morando J, Mauad MA, Piazera FZ, Souza MP De, Oliveira C, Machado C, et al. Transplante de células-tronco hematopoéticas em crianças e adolescentes com leucemia aguda . Experiência de duas instituições brasileiras. (55 41).

9. Marques M. MEDULA ÓSSEA AO DIAGNÓSTICO E AO SEGUIMENTO DO $\mathrm{DE}$ TRATAMENTO

AGUDA ( LLA ) MEDULA ÓSSEA AO DIAGNÓSTICO E AO SEGUIMENTO DO TRATAMENTO DE LEUCEMIA LINFOIDE AGUDA ( LLA ). 2013;

10. JANAÍNA DA SILVA LEMOS LEUCEMIA LINFÓIDE AGUDA : AVANÇOS NO DIAGNÓSTICO RECIFE JANAÍNA DA SILVA LEMOS LLA: AVANÇOS NO DIAGNÓSTICO. 2013;0-48.

11. Paiva R, Paulo S. Universidade de São Paulo Faculdade de Saúde Pública Infecções na infância , características maternas e leucemia linfocítica aguda em crianças Infecções na infância , características maternas e leucemia linfocítica aguda em crianças. 2014;

12. Aparecida U, Resende D, Intra R, Diagn IDOS, Morfol S, Das M, et al. UNIVERSIDADE FEDERAL DO TRIÂNGULO MINEIRO. 2013;

13. Casos OPDE, Classilcados C, Identilcar P, Celular AL. DIAGNOSTICO

LABORATORIAL DAS LEUCEMIAS LINFOIDES AGUDAS. LEUCEMIA LINFOIDE 
aguda: uma doença curável Acute lymphoblastic leukemia : a curable disease. 2012;2(1):63-8.

15. Em PDEP, Ciências M. No Title. 2010;

16. Costa L, Lichtvan L. Citogenética nas leucemias linfoides agudas. 2007;

17. Original A. AVALIAÇÃO DOS RISCOS OCUPACIONAIS DE TRABALHADORES DE SERVIÇOS DE RADIOLOGIA *. 2005;38(4):279-81.

18. Henrique $\mathrm{P}$, Visacre $\mathrm{M}$. O TRANSPLANTE AUTÓLOGO COMO FORMA DE TRANSPLANTATION FOR THE. 2010;26:35-39.

19. Siebel RS, Marchioro MK, Bueno D. Estudo de prescrições de antineoplásicos e antimicrobianos em uma unidade de oncologia pediátrica Study of antineoplastic and antimicrobial prescriptions in a pediatric oncology unit. 2012;32(3):30310.

20. Medicina FDE, Patologia MEM, Willian D, Sousa LDE. DANIEL WILLIAN LUSTOSA DE SOUSA EXPRESSÃO DA LSELECTINA E DO CD44 NAS LEUCEMIAS EXPRESSÃO DA
L-SELECTINA E DO CD44

NAS LEUCEMIAS. 2009;

21. Albertina N, Mara S, Aristides A.

A IMUNOFENOTIPAGEM NO

DIAGNÓSTICO

DIFERENCIAL DAS

LEUCEMIAS AGUDAS: UMA REVISÃO As leucemias agudas são doenças monoclonais caracterizadas pela proliferação de células hematopoiéticas anormais na medula célula progenitora do hematopoiético na medula óssea. 2008;12(1):5-14.

22. Pérez-Vera $P$, et al. Cytogenetics in acute llymphoblastic leukemia in mexican children: a institucional experience. Archivesof Medical Research. 2001;32:202-207.

23. Udayakumar A. et al. Cytogenetic profile of childhood acute lymphoblastc leukemia. Archives of Medical Research. 2007;38:305-312.

24. Lichtvan LCL. Citogenêtica nas Leucemias Linfóides Agudas. Ribeirão Preto, 2007.

25. Swaminathan R, Rama R, Shanta V. Childhood cancers in Chennai, India, 1990-2001: incidence and survival. International Journal of Cancer. 2008;122:2607-11. 
26. Ries LAG, Smith MA, Gurney JtuF, Tamra T, Young JL. Cancer incidence and survival among children and adolescents: United States SEER Program 1075 1995. Bethesda. National Cancer Institute, 1999.

27. Linet MS, Wacholder S, Zahm SH. Intepreting epidemiologic research: lessons from studies of chidhood cancer. Pediatrics. 2003;112(1):218-32.

28. Linabery AM, Ross JA. Trends in childhood cancer incidence in the USA (1992-2004). Am Cancer Society. 2008;112(2):416-32.

29. Ma SK. Clinical presentation, hematologic fratures and treatment outcome of childhood acute lymphoblastic leukemia. Hematological Oncology. 1997;15:141-49.

30. Chessells JM. Pitfalls in the diagnosis of childhood leukemia. British Journal of Hematology. 2003;114:506-11.

31. Margolin JF, et al., Acute lymphoblastic leukemia. In: Pizzo PA, Poplack DG. Principles and practice of pediatric oncology. [S.1]: Lippincott Willians and Wikins. 2005;539-590.
32. Rego EM, et al., Subpopulação linfocitárias na medula óssea normal de crianças e adultos. Significado clínico da heterogeneidade da intensidade de expressão do antífeno CD10 na leucemia linfóide aguda do subtipo comum. São Paulo, 1997.

33. Dworzak MN, Panzer-Guimayer ER. Flow cytometric detection of minimal residual diase in acute lymphoblastic leukemia, lymphoma. 2003;44:1445-1455.

34. Leite EP. Fatores prognósticos em crianças e adolescentes com leucemia linfóide aguda. Revista Brasileira de Saúde Materno Infantil, Recife. 2007;7(4):413-421.

35. Farias MG, Castro SM de. Diagnóstico laboratorial das leucemias agudas. Jornal Brasileiro de Patologias. Medicina Laboratorial. 2004;40(2):22-35.

36. Casperson $\mathrm{T}$, Zech L, Johonsson C, Modest EJ. Identification of human chromosomes by DNA - binding fluorescent agents. Chromosoma. 1970;30:2215-227.

37. Seabrigth MA. Rapid Banding Technique for Human Chromossome. Lancet. 1971;2:971972.

38. Kowalczyk JR, Babicz M, Gaworczyk A, Lejman M, Winnicka D, Sthyka 
B. Structural and numerical abnormalities resolved in one step analysis: the most common chromosomal rearranfements detected by comparative genomic hybridization in chilhood acute lymphoblastic leukemia. Cancer Genetics and Cytogenetics. 2010;200(2):161-166.
39. Silva MLM. Cytogenetics analysis of 100 consecutive newly diagnosed cases of acute lymphoblastic leukemia in Rio de Janeiro. Cancer Genetics an Cytogenetics. 2002;137:85-90. 